\title{
Intrapericardial paraganglioma: The role of integrated advanced multi-modality cardiac imaging for the assessment and management of rare primary cardiac tumors
}

\author{
Sophie Degrauwe ${ }^{1}$, Pierre Monney ${ }^{1}$, Salah D. Qanadli ${ }^{2}$, John Prior, Catherine Beigelmann- \\ -Aubry ${ }^{2}$, Pier-Giorgio Masci ${ }^{1}$, Eric Eeckhout ${ }^{1}$, Olivier Muller ${ }^{1}$, Juan F. Iglesias ${ }^{1}$ \\ ${ }^{1}$ Department of Cardiology, Lausanne University Hospital, Lausanne, Switzerland \\ ${ }^{2}$ Department of Radiology, Lausanne University Hospital, Lausanne, Switzerland \\ ${ }^{3}$ Department of Nuclear Medicine, Lausanne University Hospital, Lausanne, Switzerland
}

Cardiac paragangliomas are rare primary cardiac tumors arising from chromaffin cells of the sympathetic ganglia [1,2]. Malignant paragangliomas are extremely uncommon, but they are associated with poor clinical outcomes due to high recurrence rates [3]. Complete surgical resection is considered the most effective treatment strategy with favorable long-term outcomes [4]. However, the optimal management of cardiac paragangliomas remains a challenging clinical dilemma owing to their high propensity for local invasion and proximity to major vascular structures that may preclude a curative surgical approach and affect long-term prognosis [3]. Preoperative assessment is crucial to determine exact location, local extension, relationship with vascular structures and potential malignancy of cardiac masses. Noninvasive and invasive cardiac imaging remains therefore the mainstay of primary cardiac tumor assessment with the objective of achieving accurate preoperative diagnosis and guide the optimal management strategy. Due to a lack of randomized clinical data, the appropriate preoperative management of primary cardiac tumors remains however controversial. Echocardiography, cardiac computed tomography (CT), cardiac magnetic resonance (CMR) imaging, myocardial positron emission tomography (PET)-CT and coronary angiography separately provide complementary information, but the overall diagnostic accuracy is limited by their respective individual advantages and limitations. Comprehensive integration of advanced cardiac imaging modalities combining both morphological and functional information with the aim of guiding optimal management of rare but potentially fatal primary cardiac tumors has never been reported on to date.

Herein we report on a 43-year-old male with pituitary macroprolactinoma on long-term low-dose Cabergoline, an ergot-derived dopamine receptor agonist, who was referred to an outpatient cardiology clinic for routine transthoracic echocardiogram (TTE) to rule-out drug-induced valvular heart disease. The TTE incidentally revealed a large $75 \times 50 \mathrm{~mm}$ hyperechogenic paracardiac mass in direct contact with the basal lateral left ventricular (LV) wall and partially compressing the LV cavity without pericardial effusion. CMR imaging (Fig. 1C, G, K) demonstrated a large, unique and well-circumscribed intrapericardial mass with fine granular appearance located at the level of the basal LV lateral wall and extending towards the atrioventricular groove. Gadolinium enhanced first pass perfusion imaging demonstrated intense and homogenous signal enhancement indicating dense vascularity. Late gadolinium enhancement imaging demonstrated bright and slightly inhomogeneous enhancement with a regularly enhanced outer rim, suggesting encapsulation. The mass appeared isointense to the myocardium on T1-weighted and

Address for correspondence: Juan F. Iglesias, MD, Department of Cardiology, Lausanne University Hospital (CHUV), Rue du Bugnon 46, 1011 Lausanne, Switzerland, tel: +4121314 00 10, fax: +412131400 13,

e-mail: Juan-Fernando.Iglesias@chuv.ch

Received: 15.01.2017 Accepted: 13.04.2017 


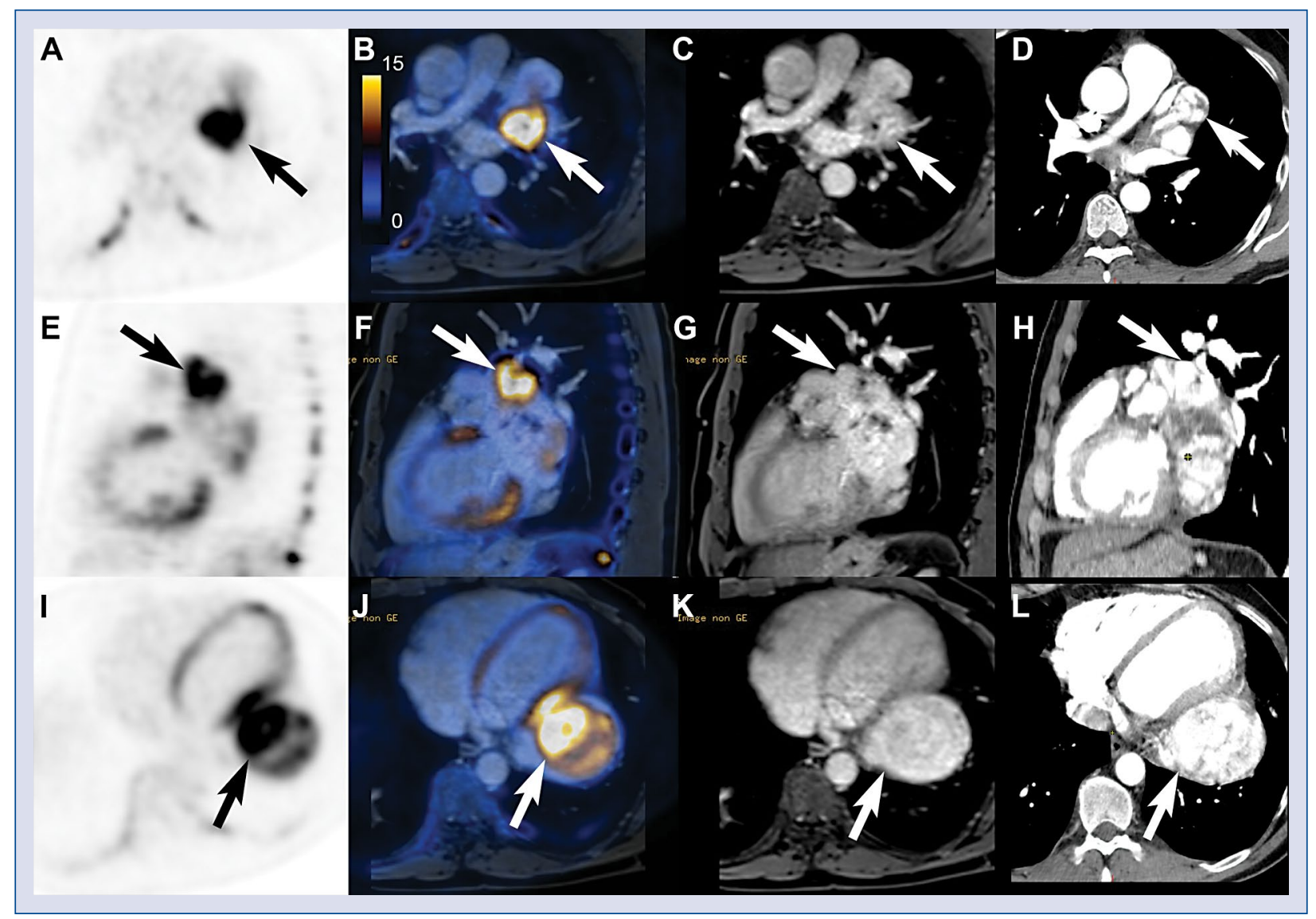

Figure 1. Integrated advanced multi-modality noninvasive cardiac imaging demonstrating transaxial (A) and sagittal (E) ${ }^{18}$ F-fluorodeoxyglucose (FDG) myocardial positron emission tomography (PET)-computed tomography (CT) scan images of paracardiac mass (black arrows) at the level of pulmonary vessels, and transaxial (I) FDG PET-CT scan image of cardiac mass (black arrow) at the myocardial level. Corresponding images of cardiac mass (white arrows) at the same levels are shown for different imaging modalities, including PET-CT scan-cardiac magnetic resonance (CMR) fusion (B, F, J), CMR (C, G, K) and contrast-enhanced CT (D, H, L).

hyperintense on $\mathrm{T} 2$-weighted images suggesting high water content or slow flowing blood. Tagged cine CMR imaging showed normal deformation of the myocardium in direct contact to the mass during systole, excluding significant myocardial invasion. Chest CT-angiography confirmed a large, heterogeneous and highly vascular paracardiac mass arising from the pericardium and compressing the lateral LV wall and the left inferior pulmonary vein without local tissue invasion (Fig. 1A, B). The blood supply to the mass originated from a tortuous and aneurysmal artery arising from the left main coronary artery (LMCA). A contrast-enhanced abdomen and pelvis CT scan confirmed the absence of distant metastases. Coronary angiogram showed a voluminous vascular paracardiac mass and defined nutrient vessel through large and tortuous arteries arising from the LMCA and the distal right coronary artery (Fig. 1C, D). Finally,
${ }^{18}$ F-fluorodeoxyglucose (FDG) PET-CT (Fig. 1A, E, I) scan showed high metabolic activity within the paracardiac mass but no hypercaptation of remote organs, excluding presence of metastases. Based on the integrated multi-modality noninvasive cardiac imaging (Fig. 1B, F, J) work-up suggestive of an encapsulated, highly vascular, and intrapericardial tumor without surrounding tissue invasion or distant metastases, surgical resection of the paracardiac mass through a median sternotomy was performed. Intraoperative inspection revealed a voluminous encapsulated mass, in close contact with the left atrial appendage, the coronary arteries and the coronary sinus, and without macroscopic invasion of the surrounding cardiac structures. The tumor was successfully resected albeit requiring prolonged cardiopulmonary bypass owing to its high bleeding tendency and close anatomic proximity to the major cardiac structures. The postopera- 
tive course was uneventful and the patient was discharged at day 6 . The postoperative TTE showed normal LV function with only a localized ex-vacuo pericardial effusion at the level of the LV lateral wall. Histopathological examination of the tumor was consistent with monomorphic cellular proliferation arranged in a Zellballen pattern surrounded by richly vascular tissue and sustentacular cells. Immunohistochemical stains were positive for neuroendocrine differentiation markers (CD56, chromogranin A, synaptophysin) and S-100. Increased plasma levels of metanephrine, normetanephrin, and 3-methoxytyramine were consistent with a functionally active cardiac paraganglioma. Genetic analysis demonstrated an heterozygous mutation in the succinate dehydrogenase subunit $\mathrm{C}$ (SDHC) encoding gene, confirming familial paraganglioma.

Cardiac paragangliomas are rare neuroendocrine tumors accounting for $<1 \%$ of all primary cardiac tumors [1]. They are mainly located in the pericardium arising from ganglia associated with the aorta and the pulmonary or coronary arteries, while only one third originate from intrinsic cardiac ganglia located within the atrial walls [4]. Malignant paragangliomas occur in only $10 \%$ of patients owing to presence of distant metastases and local tissue invasion [2], but they are associated with poor clinical outcome due to high recurrence rates [3]. Preoperative noninvasive cardiac imaging remains therefore essential, but optimal preoperative assessment and factors associated with adverse surgical and long-term outcomes are still unclear, as current evidence is limited to retrospective observational series including small number of patients. Integration and co-registration of advanced cardiac imaging modalities combining both anatomic (cardiac CT and/or CMR) and functional $\left({ }^{18} \mathrm{FDG}\right.$ PET-CT) information provide a unique combination of morphological and metabolic tumor characterization. This case highlights the incremental value of integrated advanced imaging modalities for the diagnosis and the management of rare but potentially malignant primary cardiac tumors aimed at providing a curative treatment stategy key to long-term survival.

\section{Conflict of interest: None declared}

\section{References}

1. Khan MF, Datta S, Chisti MM, et al. Cardiac paraganglioma: clinical presentation, diagnostic approach and factors affecting short and long-term outcomes. Int J Cardiol. 2013; 166(2): 315-320, doi: 10.1016/j.ijcard.2012.04.158, indexed in Pubmed: 22652039.

2. Ramlawi B, David EA, Kim MP, et al. Contemporary surgical management of cardiac paragangliomas. Ann Thorac Surg. 2012; 93(6): 1972-1976, doi: 10.1016/j.athoracsur.2012.02.040, indexed in Pubmed: 22537533.

3. Bravo EL, Tagle R. Pheochromocytoma: state-of-the-art and future prospects. Endocr Rev. 2003; 24(4): 539-553, doi: 10.1210/ /er.2002-0013, indexed in Pubmed: 12920154.

4. Plouin PF, Duclos JM, Soppelsa F, et al. Factors associated with perioperative morbidity and mortality in patients with pheochromocytoma: analysis of 165 operations at a single center. J Clin Endocrinol Metab. 2001; 86(4): 1480-1486, doi: 10.1210/ /jcem.86.4.7392, indexed in Pubmed: 11297571. 